\title{
Evaluación del cambio de uso de suelo en un humedal relicto de la costa peruana
}

Evaluation of land use change in a relict wetland on the Peruvian coast

\author{
Zaida Sanchez $^{1 *}{ }^{\oplus}$, Leslye Millones ${ }^{2} \odot$ \\ y Jazmín Ordoñez ${ }^{2} \odot$
}

1 Universidad Científica del Sur. Lima, Perú.

2 Universidad San Ignacio de Loyola. Lima, Perú.

\section{SOUTH} SUSTAINABILITY

Citar como: Sanchez, Z., Millones, L. y Ordoñez, J. (2021). «Evaluación del cambio de uso de suelo en un humedal relicto de la costa peruana». South Sustainability, 2(1), e027. doi: https://doi.org/10.21142/SS-02012021-e027

Artículo recibido: 16/10/2020 Revisado por pares Artículo aprobado: 14/1/2021

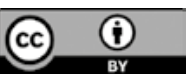

(c) Las autoras, 2021. Publicado por la Universidad Científica del Sur (Lima, Perú)

${ }^{*}$ E-mail de correspondencia: zaidasanchezlopez.zsl@gmail.com

\section{RESUMEN}

El cambio de uso de suelos es un factor influyente en la recuperación y conservación de relictos de los humedales. En este artículo se identificó y cuantificó la evolución espacio-temporal de los usos de suelo en un humedal relicto de la costa peruana, perteneciente a la zona de recuperación del sitio Ramsar Los Pantanos de Villa. Se procesaron imágenes satelitales en periodos de cada cuatro años (2001-2021), a través de la clasificación supervisada en sistemas de información geográfica y reconociendo los usos de suelos según la densidad de la cobertura vegetal, las zonas urbanas y los cuerpos de agua. Las tasas de variación 2001-2021 muestran la pérdida progresiva de vegetación densa e intermedia con un $-55,30 \%$ y $-11,54 \%$, respectivamente. Asimismo, es posible apreciar el crecimiento de las zonas sin vegetación y zonas urbanas en un 50,62 \% y 474,21 \%, respectivamente. Estos resultados se relacionan con los cambios identificados en el campo, como la introducción de animales de pastoreo, la disposición de efluentes domésticos, la disposición y quema de residuos municipales y la apertura de vías de comunicación. Estos dos últimos cambios son las causas directas de la pérdida de cobertura vegetal y el aumento en la propagación de enfermedades. Se concluye que la gestión de los puntos de acopio de residuos municipales y el acceso al servicio de agua y desagüe son de vital importancia para garantizar la conservación del relicto y para mejorar la calidad de vida de la población aledaña.

\section{Palabras clave: uso de suelo, clasificación supervisada, relicto, humedal}

\section{ABSTRACT}

Land use change is an influential factor in the recovery and conservation of relict wetlands. In this article, the spatial and temporal evolution of land use was identified and quantified in a relict wetland on the Peruvian coast, forming part of the recovery zone of the Pantanos de Villa Ramsar site. Satellite images were processed every four years (from 2001 to 2021) through supervised classification of geographic information systems, identifying land use based on the density of vegetation cover, urban areas and bodies of water. The 2001-2021 variation rates demonstrate the progressive loss of dense and intermediate vegetation by $-55.30 \%$ and $-11.54 \%$, respectively. Also, the expansion was identified of areas without vegetation and urban areas, by $50.62 \%$ and $474.21 \%$ respectively. These results are related to changes identified in the field, such as the introduction of grazing animals, the disposal of domestic effluent, the disposal and burning of municipal waste, and the opening up of communication routes, with these last two factors constituting the direct causes of loss of vegetation cover and the increase in the spread of disease. In conclusion, management of municipal waste collection points and access to water and sewage services are of vital importance in order to ensure conservation of the wetland relict and improve the quality of life of the neighboring population.

Keywords: Land use, supervised classification, relict, wetland 


\section{Introducción}

Actualmente, el crecimiento de las zonas urbanas ha sobrepasado los sitios originales de las ciudades, y abarcan espacios cada vez más extensos y de manera desordenada (Amaya, 2005). Los humedales relictos presentan diversas amenazas. La principal es el cambio de uso de suelo, que modifica la cubierta vegetal y lo convierte a hábitats naturales remanentes (Cárdenas y Hurtado, 2019). El 75 \% de los humedales costeros de Lima tienen una afectación directa por el crecimiento demográfico y el desarrollo urbanístico (Aponte et al., 2020). En este sentido, los Pantanos de Villa se encuentran bajo presiones diferentes a las naturales, como urbanización, degradación y reducción de la densidad de la vegetación por prácticas como la agricultura, ganadería y pastoreo, incendios, acumulación de residuos sólidos, entre otros (Aponte et al., 2020). Por estas fuentes de cambio directo, en 2018 se identificó una reducción del área vegetal en Pantanos de Villa de un $18 \%$, tomando a 2008 como año base (Flores et al., 2020).

Las actividades humanas que se desarrollan dentro y en zonas relicto de los alrededores del Área Natural Protegida (ANP) de Los Pantanos de Villa afectan el suelo dentro de ella y generarán impactos como la afectación de las condiciones de calidad ambiental del humedal, el

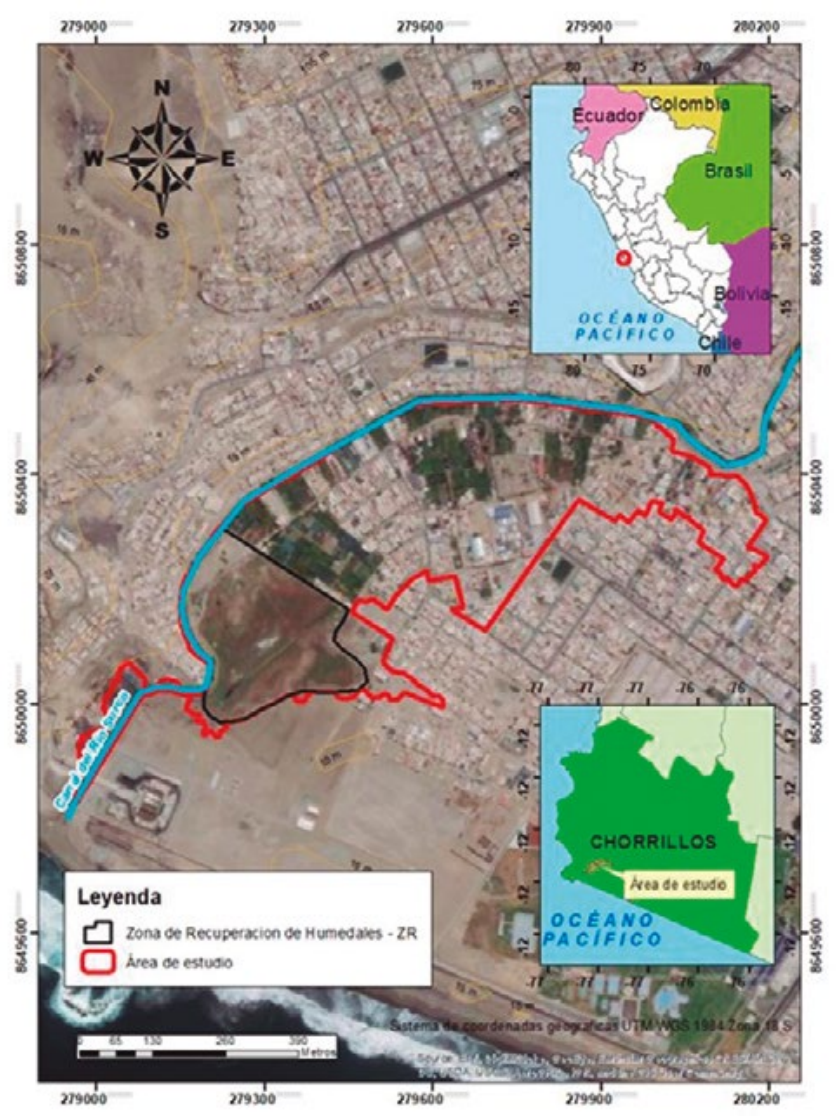

Figura 1. Zona de estudio delimitada en el relicto La Chira. Elaboración propia. alejamiento de especies, la reducción y el cambio de la cubierta vegetal (Miloslavich, 2013; Ramirez y Cano, 2010).

Ante esto, la siguiente investigación busca conocer la evolución de los últimos 20 años del uso del suelo en el relicto de humedal en Los Pantanos de Villa, ubicado en La Chira. Para ello se pretende identificar el uso de los suelos en este espacio y su entorno, identificar las actividades económicas que se desarrollan en el relicto y su entorno, y determinar la situación actual en el uso de los suelos en esta zona de estudio. Este trabajo contribuiría con el objetivo estratégico "Supervisión y vigilancia del cumplimiento de medidas ambientales dentro de la Zona de Reglamentación Especial».

\section{Materiales y métodos}

\section{Área de estudio}

El relicto de La Chira se encuentra a 3,34 km al noroeste del ANP Los Pantanos de Villa, en la extensión de la planta de tratamiento de aguas residuales de La Chira. El área trazada geográficamente por la Autoridad Municipal de los Pantanos de Villa (PROHVILLA) se considera como $Z R$, presente al lado del río Surco, que desemboca en el océano Pacífico (figura 1). Existen centros poblados alrededor: el asentamiento humano Hijos de Miyashiro y el asentamiento humano Colinas del Pacífico ubicados al norte, el asentamiento humano Pacífico de Villa ubicado al oeste, y el asentamiento humano Bello Horizonte al este.

La ZR en La Chira presenta un área total de 8,78 hectáreas y el área de estudio trazada presenta un área de 30,3 hectáreas.

Las temperaturas de la zona una temperatura promedio de $15{ }^{\circ} \mathrm{C}$ a $26{ }^{\circ} \mathrm{C}$. El periodo de llovizna, aunque muy leve, se da entre junio y septiembre (ParksWatch, s. f.).

Tabla 1. Conjunto de datos usados en el estudio en los humedales relicto de Chira.

\begin{tabular}{|c|c|c|c|}
\hline 巳ั & $\begin{array}{l}\text { Fecha } \\
\text { (mes/día/ } \\
\text { año) }\end{array}$ & Resolución & $\begin{array}{c}\text { Cobertura en km } \\
\text { (parte superior } x \text { izquierda } \times \\
\text { derecha } \times \text { parte inferior) }\end{array}$ \\
\hline \multirow{6}{*}{ 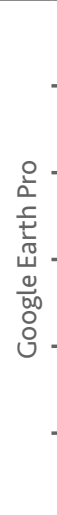 } & $5 / 4 / 2001$ & $0,41 \times 0,41 \mathrm{~m}$ & $\begin{array}{c}8650,652 \times 278,561 \times 280,549 \times \\
8649,736\end{array}$ \\
\hline & $2 / 26 / 2005$ & $0,41 \times 0,41 \mathrm{~m}$ & $\begin{array}{c}8650,652 \times 278,561 \times 280,549 \times \\
8649,736\end{array}$ \\
\hline & $1 / 24 / 2009$ & $0,41 \times 0,41 \mathrm{~m}$ & $\begin{array}{c}8650,652 \times 278,561 \times 280,549 \times \\
8649,736\end{array}$ \\
\hline & 2/13/2013 & $0,41 \times 0,41 \mathrm{~m}$ & $\begin{array}{c}8650,652 \times 278,561 \times 280,549 \times \\
8649,736\end{array}$ \\
\hline & 2/21/2017 & $0,41 \times 0,41 \mathrm{~m}$ & $\begin{array}{c}8650,652 \times 278,561 \times 280,549 \times \\
8649,736\end{array}$ \\
\hline & $4 / 23 / 2021$ & $0,41 \times 0,41 \mathrm{~m}$ & $\begin{array}{c}8650,652 \times 278,561 \times 280,549 \times \\
8649,736\end{array}$ \\
\hline
\end{tabular}




\section{Métodos}

Los datos usados para identificar el uso del suelo y sus cambios fueron imágenes satelitales de Google Earth Pro (tabla 1), debido a que el área de estudio es muy pequeña para usar imágenes satelitales de más de $30 \mathrm{~m}$ de resolución espacial, como las Landsat 8 . Google Earth Pro tiene disponibles imágenes en alta resolución de múltiples satélites desde 2001 hasta el presente, por lo que su uso es una opción (Hritz, 2013; Malarvizhi et al., 2016; Li et al., 2020). Para determinar el área de estudio se trazó el recorrido de la cobertura vegetal densa e intermedia del menor año tomado en este estudio, 2001. Debido a que las imágenes satelitales escogidas no presentaban nubes ni sombras que pudieran entorpecer el proceso, en ArcMap no se hizo ninguna corrección en ellas.

Para la verificación de las imágenes satelitales y la determinación de las actividades antropogénicas se realizaron cinco visitas a campo. En cada visita se tomaron fotos de cada foco de contaminación o degradación de suelo de cualquier tipo, como el lugar de pastoreo y la acumulación de residuos sólidos; además, se tomaron fotos de cada clase de uso de suelo determinada.

\section{Realización de mapas}

Para la determinación de las clases de uso de suelo en la realización de mapas se usó bibliografía y se realizaron

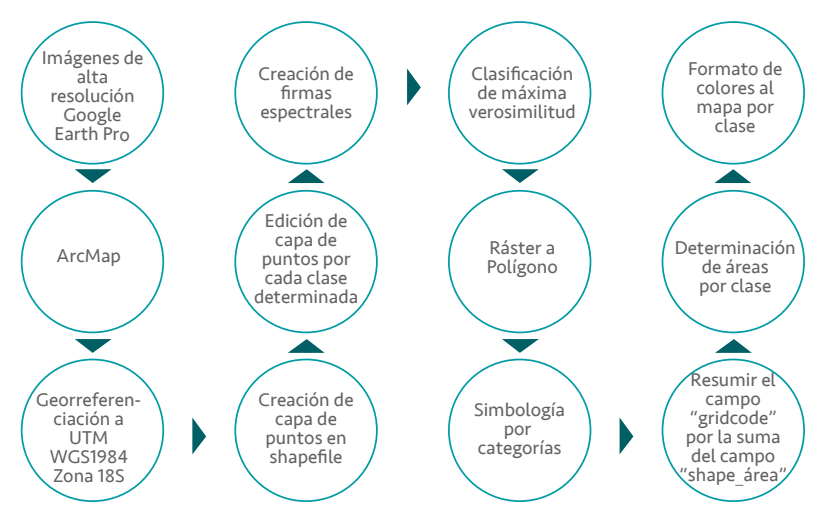

Figura 2. Proceso operacional para generar mapas de uso de suelo por año del relicto La Chira. visitas a campo (Moscoso, 2007; Pauleit et al., 2005). Posteriormente, para la realización de los mapas en ArcMap se llevó a cabo el proceso de la figura 2.

\section{Resultados}

\section{Análisis de clasificación supervisada}

Los resultados obtenidos de las áreas del relicto La Chira se encuentran en la tabla 2 y en las figuras 3 y 4 . Se aprecia que la densidad de la vegetación en los 20 años de estudio se redujo hasta ocupar un $26 \%$ del total de la superficie en estudio, la cual incluye la vegetación de la actividad agrícola para todos los años de evaluación. Por otro lado, la vegetación intermedia llegó a abril de 2021 con un aproximado del $19 \%$ del área; no obstante, en 2009 tuvo su registro más alto, al ocupar aproximadamente un $39 \%$. Ello podría asociarse a una mayor actividad de pastoreo evidenciada en la figura 5 C, ya que ese mismo año la clase de cobertura más densa ocupó aproximadamente el $24 \%$.

El crecimiento progresivo de las zonas urbanas en relación con la superficie total ha tenido un efecto en la extensión de las áreas sin vegetación. Esto se debe a

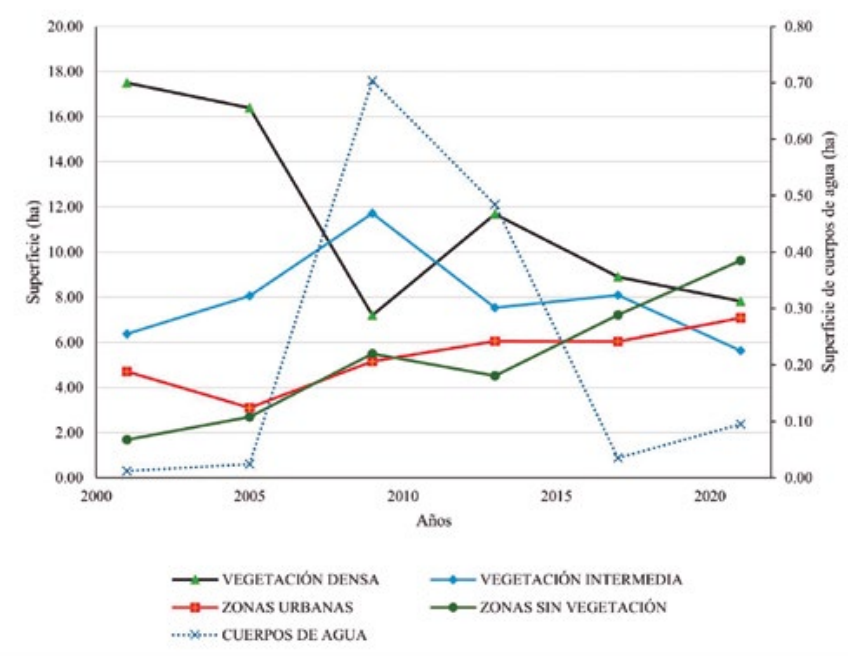

Figura 3. Evolución de la superficie del espacio temporal del uso de suelos y superficie de cuerpos de agua en el humedal de relicto La Chira.

Tabla 2. Área en hectáreas de la clasificación supervisada para el humedal de relicto La Chira en el periodo $2001-2021$.

\begin{tabular}{|c|c|c|c|c|c|c|c|c|c|c|c|}
\hline AÑo & VD & $\%$ & VI & $\%$ & $\mathbf{Z U}$ & $\%$ & ZSV & $\%$ & CA & $\%$ & TOTAL \\
\hline 2001 & 17,50 & $57,84 \%$ & 6,37 & $21,05 \%$ & 4,70 & $15,53 \%$ & 1,68 & $5,54 \%$ & 0,01 & $0,04 \%$ & 30,26 \\
\hline 2005 & 16,39 & $54,16 \%$ & 8,05 & $26,61 \%$ & 3,11 & $10,27 \%$ & 2,69 & $8,88 \%$ & 0,02 & $0,08 \%$ & 30,26 \\
\hline 2009 & 7,19 & $23,77 \%$ & 11,72 & $38,71 \%$ & 5,16 & $17,05 \%$ & 5,49 & $18,14 \%$ & 0,70 & $2,32 \%$ & 30,26 \\
\hline 2013 & 11,68 & $38,60 \%$ & 7,53 & $24,88 \%$ & 6,05 & $20,00 \%$ & 4,51 & $14,92 \%$ & 0,48 & $1,60 \%$ & 30,26 \\
\hline 2017 & 8,90 & $29,41 \%$ & 8,09 & $26,72 \%$ & 6,03 & $19,91 \%$ & 7,22 & $23,85 \%$ & 0,03 & $0,12 \%$ & 30,26 \\
\hline 2021 & 7,82 & $25,85 \%$ & 5,64 & $18,63 \%$ & 7,08 & $23,39 \%$ & 9,63 & $31,82 \%$ & 0,09 & $0,31 \%$ & 30,26 \\
\hline $\mathrm{TV}_{01-21}$ & & $-55,30 \%$ & & $-11,54 \%$ & & $50,62 \%$ & & $474,21 \%$ & & $686,97 \%$ & \\
\hline
\end{tabular}

\%: porcentaje al total de la superficie de estudio; TV 01-21: tasa de variación en el periodo 2001-2021; VD: vegetación densa; VI: vegetación intermedia; ZU: zonas urbanas; ZSV: zonas sin vegetación; CA: cuerpos de agua. 

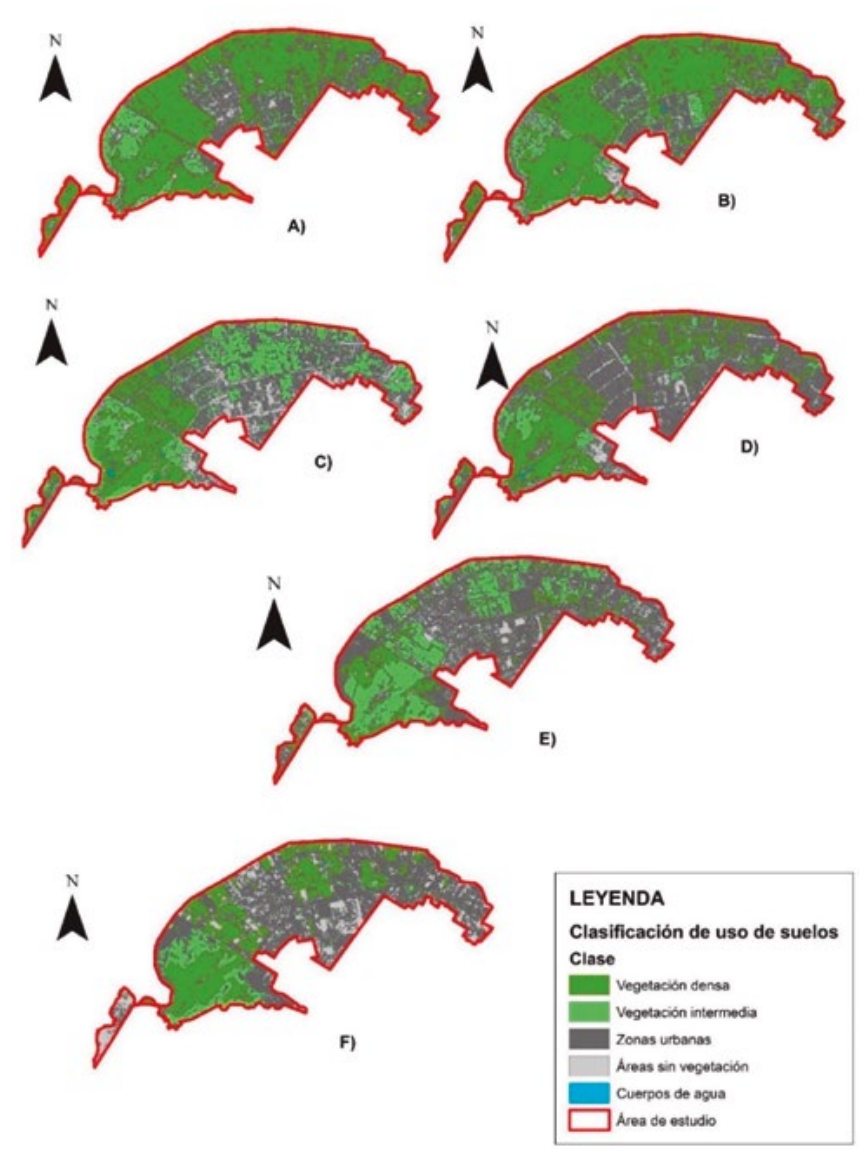

Figura 4. Mapas de evaluación espacio-temporal del uso de suelos de la zona de estudio. A) 2001, B) 2005, C) 2009, D) 2013, E) 2017, F) 2021.

los requerimientos urbanos para vivienda y movilidad, además de otros flujos básicos para el sustento, por lo que ha aumentado un 474,21\% el área sin vegetación.

Finalmente, la extensión de los cuerpos de agua para el relicto en estudio tuvo un crecimiento de casi ocho veces de la superficie inicial. Sin embargo, en 2009 y 2013 se encuentran los niveles más altos, los cuales se pueden asociar a un error en la clasificación supervisada, la variación de las condiciones del entorno y la carga hídrica de los canales. Estos dos últimos aspectos no cuentan con un adecuado sistema de drenaje de efluentes domésticos y algunas veces la carga hídrica es dispuesta en la vía pública (figura 5), por lo que esta carga hídrica mencionada puede ser clasificada también como cuerpos de agua por la clasificación supervisada. Cabe precisar que, al emplearse imágenes satelitales de los veranos de cada año (con excepción de 2021), las condiciones de carga hídrica pueden tener mayor variación.

\section{Análisis de visitas de campo}

La figura 5 resume los principales hallazgos de la visita de campo, donde se encontraron espacios con vegetación intermedia y parches sin vegetación, además de tener los espejos de agua secos, que dejaban a la vista residuos sólidos. Se evidenció también actividad de pastoreo en la zona del relicto (figura 5 C). Asimismo, al no contar con una cantidad de puntos de acopio de residuos sólidos autorizados que abastezca a la población establecida en la zona, es posible observar acumulación de residuos domésticos en el canal y en el contorno del relicto (figura $5 \mathrm{D}$ y $5 \mathrm{E}$ ). Cabe precisar que los centros poblados ubicados alrededor no disponen del servicio de agua y desagüe, razón por la cual se ven en la necesidad de conducir sus efluentes por medio de tuberías y mangueras hacia el exterior de las viviendas.

Además, por el desarrollo de viveros es posible identificar residuos de la actividad, los cuales tienden a ser incinerados en espacios del relicto (figura $5 \mathrm{G}$ ), lo que afecta las condiciones del suelo, sin considerar la generación de emisiones y el grado de afectación en las condiciones del canal del río Surco.

En cuanto al desarrollo urbano, siendo esta una clase establecida en la clasificación supervisada planteada en el estudio, es posible justificar el resultado en el área planteada, dada la extensión presente en el noroeste del relicto (figura $5 \mathrm{H}$ ).

\section{Discusiones}

El área más afectada por los cambios en el uso de los suelos es aquella compuesta por la vegetación densa; las otras categorías han ocupado parte de lo que esta cubría en 2001. De acuerdo con el Sistema Nacional de Información Ambiental (SINIA), la pérdida de cobertura vegetal para ecosistemas costeros y andinos tuvo un promedio de 269,84 hectáreas en el periodo 2010-2018 (DCOTA, 2018). El efecto del cambio de uso de suelos también se manifiesta en la fluctuación de la napa freática, que desciende a causa de la ausencia de la filtración natural (Pulido y Bermúdez, 2018).

El descenso de la vegetación densa se relaciona probablemente con la ausencia de normativa que reglamente las actividades del ganado vacuno y porcino de zonificación del distrito (actividad que no se encuentra en el índice para la ubicación de actividades urbanas en la Ordenanza Municipal 620-MML) (PROHVILLA, 2015; Chorrillos, 2019). Un caso similar ocurre en los humedales Chochoc y de Valle del Cauca, donde la actividad agrícola, la urbanización y la disposición de residuos sólidos son los factores más influyentes en la degradación de la cobertura vegetal y la desaparición de especies pioneras. Este segundo problema es el más evidente, ya que presenta una reducción del 81,7 \% al año 2010 (Ruiz-Santillan et al., 2019; Pastrana y Pacheco, 2010).

El crecimiento urbano también implica un mayor uso de las necesidades básicas como el agua y desagüe, que tienen un efecto directo en las condiciones de saturación del suelocausada por los efluentes domésticos. Ello llevaría a un posible incremento del riesgo a contraer patologías 


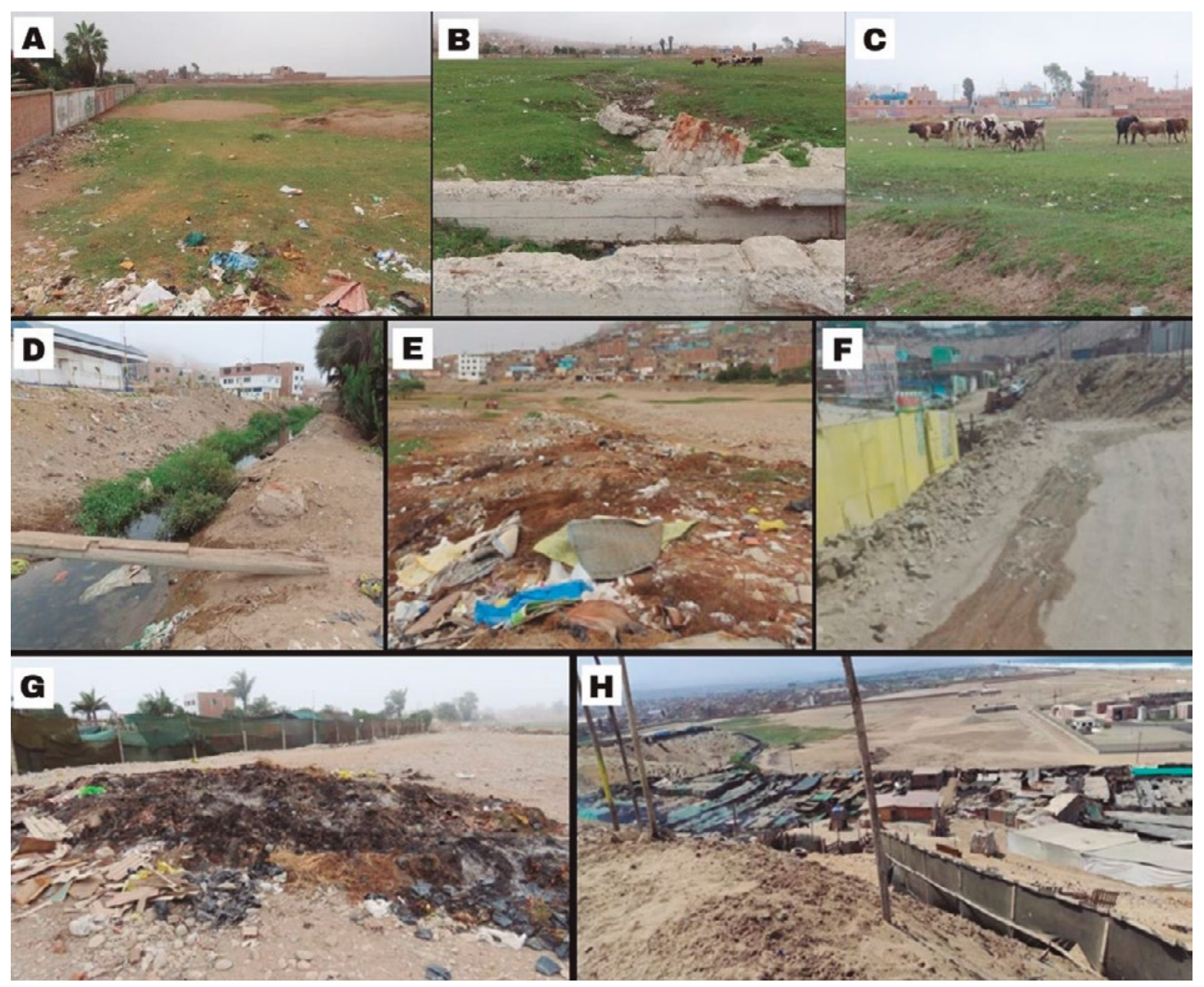

Figura 5. Estado del relicto de humedal La Chira en marzo 2021. A: extremo de la zona del relicto de humedal con Bello Horizonte; B: canal de espejo de agua del relicto; C: actividad de pastoreo; D: residuos sólidos en canal; E: residuos sólidos en el contorno; F: efluentes domésticos; G: residuos de viveros aledaños; H: asentamientos humanos aledaños.

infecciosas, que pueden exponer a todo ciudadano a microorganismos como Escherichia coli, Salmonella spp., coliformes fecales, entre otros que se proliferan en esas condiciones (Moreno y Alarcón, 2010).

En conclusión, bajo la clasificación supervisada se identificó la evolución del uso de suelos, considerando la cobertura vegetal y su densidad, además de las zonas urbanizadas y cuerpos de agua. De ello, se obtuvo un efecto negativo en la superficie de la vegetación densa y una pérdida del 55,30\% del área inicial correspondiente a 2001. Por otro lado, el crecimiento a 2021 ha sido del 50,62 \% para las zonas urbanas y está directamente relacionado con el descenso anterior. En las áreas sin vegetación la tasa aumentó $474,21 \%$ la superficie inicial debido a la disposición de residuos sólidos domésticos, pequeñas empresas en la zona dedicadas a la agricultura (viveros) y al pastoreo por la crianza de ganado vacuno en la zona. Se concluye finalmente que la gestión de los puntos de acopio de residuos municipales y acceso al servicio de agua y desagüe son de vital importancia para garantizar la conservación del relicto y para mejorar la calidad de vida de la población aledaña.

\section{Agradecimientos}

Agradecemos a los profesores Héctor Alonso Aponte Ubillús y Luis Alfredo Roca Infante por brindarnos el apoyo en esta investigación.

\section{Contribución de la autoría}

ZS se encargó de escoger y realizar la metodología adecuada y redacción de una corta parte del manuscrito; LM se encargó de gran parte de la redacción del manuscrito, evaluación y realización de los gráficos; y JO, de mediana parte de la redacción del manuscrito.

\section{Conflicto de intereses}

El presente estudio no presenta conflicto de intereses. 


\section{Referencias bibliográficas}

Amaya, C. (2005). «El ecosistema urbano: simbiosis espacial entre lo natural y lo artificial». Revista Forestal Latinoamericana, 37, pp. 1-16.

Aponte, H., Gonzales, S. y Gomez, A. (2020). «Impulsores de cambio en los humedales de América Latina: el caso de los humedales costeros de Lima». South Sustainability, 1(2), p. 3.

Cárdenas, W. N. y Hurtado, L. B. (2019). «Variación de la abundancia y diversidad de aves en el Humedal Lucre-Huacarpay, Quispicanchi / Cusco / Perú, durante el periodo de "El Niño" 2015-2016». Ecología Aplicada, 18(2), p. 111.

Chorrillos, 2019. «Plano de zonificación». Disponible en: http://www. munichorrillos.gob.pe/sites/default/files/documentos/desarrollourbano/plano-de-zonificacion-mdch-2019.pdf

DGOTA, 2018. Sistema Nacional de Información Ambiental. Disponible en: https://sinia.minam.gob.pe/indicadores/listado

Flores, N., Castro, I. y Aponte, H. (2020). «Evaluación de las unidades de vegetación en los Pantanos de Villa (Lima, Perú) mediante Sistemas de Información Geográfica y Teledetección». Arnaldoa, 27(1), p. 10.

Hritz, C. (2013). «A malarial-ridden swamp: using Google Earth Pro and Corona to access the southern Balikh valley, Syria». Journal of Archaeological Science, 40(4), pp. 1975-1987.

Li, W. et al. (2020). «Integrating Google Earth imagery with Landsat data to improve 30-m resolution land cover mapping». Remote Sensing of Environment, 237, 111563.

Malarvizhi, K., Vasantha Kumar, S. y Porchelvan, P. (2016). «Use of high resolution Google Earth satellite imagery in landuse map preparation for urban related applications». Procedia Technology, 24, pp. 1835-1842.
Miloslavich, P. (2013). Variación y protección de humedales costeros frente a procesos de urbanización: casos Ventanilla y Puerto Viejo. Tesis de magíster en Desarrollo Ambiental. Pontificia Universidad Católica del Perú.

Moreno, M. y Alarcón, A. (2010). «Higiene alimentaria para la prevención de trastornos digestivos infecciosos y por toxinas». Revista Médica Clínica Las Condes, 21(5).

Moscoso, C. (2007). Cambios en los usos y coberturas de suelo y sus efectos sobre la escorrentía urbana. Valparaíso y Viña del Mar, Período 1980-2005. Tesis de grado en Geografía. Universidad de Chile.

Parks Watch. (2004). Parks Watch. Disponible en: http://parkswatch. org/parkprofile.php?l=spa\&country=per\&park=pvrz\&page=phy

Pastrana, E. y Pacheco, Y. (2010). La Convención Ramsar a lo largo del eje local-global: protección de humedales en el Valle del Cauca. Papel Político, 15(2).

Pauleit, S., Ennos, R. y Golding, Y. (2005). «Modeling the environmental impacts of urban land use and land cover change -a study in Merseyside, UK». Landscape and Urban Planning, 71, pp. 295-310.

Autoridad Municipal de los Pantanos de Villa, PROHVILLA. (2015). Base legal. Disponible en: http://prohvilla.munlima.gob.pe/admin/ archivospdf/ordenanza\%20620.pdf

Pulido, V. y Bermúdez, L. (2018). «Estado actual de la conservación de los hábitats de los Pantanos de Villa, Lima, Perú». Arnaldoa, 25(2).

Ramirez, D. W. y Cano, A. (2010). «Estado de la diversidad de la flora vascular de los Pantanos de Villa (Lima-Perú): State of vascular flora diversity from Pantanos de Villa (Lima-Peru)». Revista Peruana de Biología, 17(1), pp. 111-114.

Ruiz-Santillan, M., Huamán, E. y Mejía, F. (2019). «Diagnóstico ecológico del Humedal Chochoc». Rebiol, 39(2), p. 10. 